\title{
Key Contributing Factors to the Acceptance of Agents in Industrial Environments
}

\author{
Stamatis Karnouskos, Senior Member, IEEE, and Paulo Leitão, Senior Member, IEEE
}

\begin{abstract}
Multiple software agent-based solutions have been developed during the last decades, and applied with varying success to different domains offering control, reconfiguration, diagnosis, monitoring, etc. However, the promise that they once posed in terms of a new alternative decentralized approach offering modularity, flexibility and robustness, is only partially fulfilled. This paper investigates some key factors, i.e., design, technology, intelligence/algorithms, standardization, hardware, challenges, application and cost, which are hypothesized to be linked to the Industrial Agent acceptance. Empirical data was acquired via a conducted survey, and statistically analyzed to investigate the support of the posed hypotheses. The results indicate that all the factors are seen important issues that play a role toward deciding for or against an industrial agent solution.
\end{abstract}

Index Terms-Cyber-physical systems, industrial agents, industrial acceptance, multiagent systems (MAS), survey.

\section{INTRODUCTION}

$\mathbf{M}$ ULTIAGENT systems (MAS) [1] is a suitable technology to develop modular, flexible, robust, and adaptive complex (large-scale) systems, based on the decentralization of functions (e.g., control, reconfiguration, diagnosis, monitoring etc.) over a community of distributed, autonomous, and cooperative agents [2], [3]. The application of agent technology allows the achievement of important features, namely modularity, flexibility, robustness, adaptability, reconfigurability, and responsiveness. The software agents' concept, established in the early 1990s, has attracted the attention of academia and industry communities, as reflected in the significant number of conferences and workshops organized around the world. These concepts were initially applied to business, electronic commerce, and management systems, but its application to industrial domains, such as manufacturing, logistics, telecommunications, smart grids and healthcare, have also been reported in several surveys [2], [4]-[9].

Industrial Agents share the common ground with software agents, inheriting their characteristics such as intelligence,

Manuscript received September 21, 2015; revised April 9, 2016, August 1, 2016, and August 7, 2016; accepted September 2, 2016. Date of publication September 8, 2016; date of current version April 18, 2017. Paper no. TII-15-1453. (Corresponding author: S. Karnouskos.)

S. Karnouskos is with SAP, Karlsruhe 76131, Germany (e-mail: stamatis.karnouskos@sap.com).

P. Leitão is with the Polytechnic Institute of Bragança, Bragança 5300, Portugal, and also with LIACC - Artificial Intelligence and Computer Science Laboratory, Porto 4150-181, Portugal (e-mail: pleitao@ ieee.org).

Color versions of one or more of the figures in this paper are available online at http://ieeexplore.ieee.org.

Digital Object Identifier 10.1109/TII.2016.2607148 autonomy, and cooperation, but their application domains are the industrial environments and applications and, therefore, need to adhere to industrial requirements [2], [6], [9]-[11]. Examples of such requirements may comprise the specific hardware integration, reliability, fault-tolerance, scalability, industrial standard compliance, quality assurance, resilience, manageability, and maintainability. Depending on the application scenarios, these requirements may have different degrees of importance and the focus is on well established, stable and proven approaches rather than experimental and not fully-tested features [6]. In any utilization scenario, Industrial Agent solutions need to fully comply with enterprise operational context and guarantee the business continuity.

In the last years, several efforts were performed to advance the domain of agents and more specifically introduce them in the real-world industrial settings. For example, in 2005, the Agentlink III coordination action for agent-based computing, funded by the European Commission, promoted a strategic roadmap in agent technology aiming to analyze the past and current states of agent technologies, and to identify the challenges and obstacles that need to be tackled for the higher commercial adoption of the technology [12]. In Germany, the VDI/VDE GMA Technical Committee 5.15 on Multiagent Systems also surveyed the use of MAS in industrial automation [11], [13].

Under the scope of various other projects, such as [2], [6], [8], [11], [14], agents prototypes that have been realized and operated in industrial environments were surveyed. Despite the rigorous research investigations and developed prototypes, a breakthrough and wide-spread usage in operational industrial settings were not achieved. Ten years ago, examples of industrial applications were rare and the implemented functionalities were highly restricted [15]. The Agent Link roadmap [12] confirmed that at the time the survey was carried out (in 2005), the agent technology was still in its infancy, both in terms of technology maturity and also in the programing skills widespread among commercial software developers. Today, after more than a decade from the first industrial application of agent technology, namely in a production line for producing cylinder heads for diesel engines at the German Daimler-Chrysler factory plant in Stuttgart [16], the progress has been at best incremental.

Key initiatives, such as Industrie 4.0 [17] and Industrial Internet [18], are emerging and feature Cyber-Physical Systems (CPS) as a key element for their success. The emergence of CPS in the last few years, and their prevalence in industrial domains [14], may provide a new opportunity for Industrial Agents [2], [19]. The reason may be associated with the software agent and especially MAS characteristics, which are envisioned to be featured by CPS at large. 
Although in the last few years, more industrial applications of agent technology are witnessed, resistance for a wider adoption by the industrial decision makers still exists, and the level of adoption expected and projected ten years ago, remains unattained today. The pertinent issue that arises from this discussion is to try to understand why, after more than ten years, the agent technology is not more widely adopted in industrial environments. It needs to be noted that in some areas, agents have already a decent adoption, but only a limited utilization of agent technology in industrial domain is reported in the literature. Therefore, this paper focuses on the factors that may impact the Industrial Agent acceptance.

In the literature, several survey review papers were published analyzing the level of adoption of agent technology in industrial environments (see, e.g., [2]-[8], [15], [19]-[26]). These review papers surveyed a set of agent-based solutions deployed in industry and concluded that very few agent systems were really built for industry. These survey papers also reflect and discuss some potential road blockers for a wider adoption by industry stakeholders. However, all these efforts do not investigate in a quantifiable manner the link between the factors that constrain the wider agent acceptance in industrial environments. This work extends and complements these efforts, by investigating and assessing empirically via a survey, the impact of key factors to Industrial Agent acceptance. This surveyed data considers the valuable knowledge in Industrial Agents domain by experts coming from industry and academia.

This paper is organized as follows: Section II discusses the theoretical aspects behind the selection of the factors to be analyzed in the survey and Section III presents the data used to perform the survey. Section IV introduces the empirical data analysis, and Section V discusses the achieved results and extracts proper observations. Finally, Section VI rounds up the paper with the conclusion.

\section{IDENTIFICATION OF KEY FACTORS}

Several surveys [4]-[7], [15], [19], performed in the last years, have tried to understand the possible barriers that are blocking the wider adoption of agent technology in industrial environments. Among others issues, these surveys have identified several important barriers, namely the demand of industry for proven and mature technologies, the initial investment, the industrial standard compliance, the missing development methodologies, the scalability and interoperability, and the integration of physical automation devices.

Based on the literature review, on the experience of the authors in developing Industrial Agent-based solutions, as well as discussions within the IEEE IES technical committee on Industrial Agents (tcia.ieee-ies.org), a set of key factors have been identified as causes that may have an impact on the Industrial Agent acceptance. Grouping common issues, the following factors have been identified (as illustrated in Fig. 1): design, technology, intelligence/algorithms, hardware, cost, standardization, application, and challenges. These key factors are seen as relevant for the decision makers when they consider the adoption of Industrial Agent solutions.

The hypotheses posed in this paper, and marked as H1-H8 in Fig. 1, claim that the respective factors may have a positive contribution to the Industrial Agent acceptance, if adequately

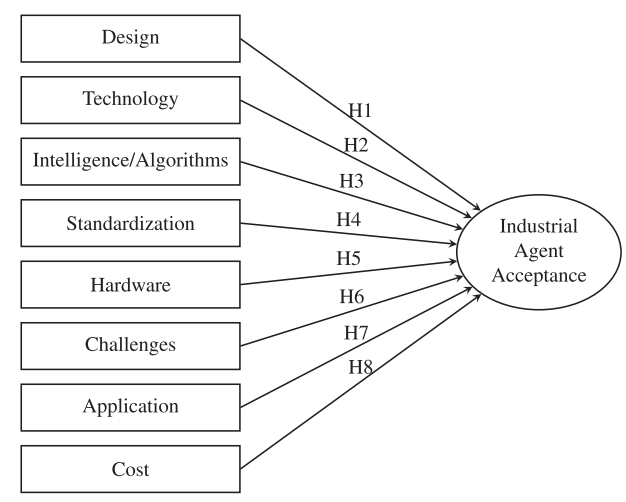

Fig. 1. Proposed model with key factors and hypotheses.

tackled. The proposed model, based on the identified hypotheses for the key factors that may impact the Industrial Agents acceptance, is empirically validated with data obtained by the survey which are subsequently statistically analyzed.

\section{A. Design}

Industrial Agents offer a new and alternative way to design complex flexible, robust, and reconfigurable systems based on the decentralization of functions. This implies a paradigm shift requiring the distributed thinking as a new way to design these systems, which may pose a possible barrier for decision makers and developers [5]. Industrial agent systems should have clear proposition on the functionalities, services, and (measurable) value-added aspects that they can offer, but not necessarily focus solely on the technical aspects. This is seen as important as decision makers are reluctant of accepting unpredictable emergent behaviors that can appear in these systems [24]. The design of industrial solutions should ensure the achievement of important properties, namely security, business continuity, scalability, reliability, robustness, fault tolerance, modularity, and interoperability [4], [21], [24]. In particular, the interoperability may be a key issue in the development of distributed and heterogeneous systems which also characterize industrial systems [4]. For this purpose, the use of open-standard interfaces in the design of industrial agent-based systems may be important for the decision-makers acceptance. The existence of a comparative methodology analysis [27], as well as best practices [7] for demonstrators running in industry that show the applicability of agents principles, may also play an important role.

\section{B. Technology}

Industrial decision makers demand solutions developed using proven, mature, and standardized technologies. As such, industrial players prefer well proved and mature technologies [4], [5], [9], instead of being the first to use them, i.e., reaching higher Technology Readiness Levels, namely levels 8 (i.e., system complete and qualified) and 9 (i.e., actual system proven in operational environment). The adoption of open technologies instead of proprietary technologies and the use of multiple heterogeneous technologies may be of importance in order to support the expertise of the developers as well as a large footprint within an organization. A look at the recent Gartner's hype cycle for emerging technologies [28] reveals several agent-related 
technologies in various stages, such as advanced analytics with self-service delivery, Internet of Things (IoT) and IoT platforms, digital security, hybrid cloud computing, machine learning, autonomous vehicles, smart advisors, and smart robots. Hence, Industrial Agents contributing and benefiting from these technologies, seem to have significant potential in the mid (5-10 years) and long term (10+ years).

The integration with legacy systems is a very important factor to support the demand for smooth migration, due to the inexistence of methods for wrapping legacy systems by agents in an automatic manner [21]. In fact, most integration efforts of agent-based applications with legacy systems was at a database level and not with planning or scheduling applications [29]. However, also other efforts have concentrated on CPS integration and simulation of service-ready devices [30], [31]. The availability of methodologies that simplifies the engineering of agent-based systems (integrating the design, analysis, simulation, and deployment phases) is seen also as highly relevant [5], [15]. Of key importance is the capability to offer to developers scalable and robust development platforms addressing the industrial requirements [4], [15]). In addition to the development platforms, the use of modern Integrated Development Environments tools may simplify the development, testing, and deployment in industrial environments.

\section{Intelligence/Algorithms}

Industrial Agents are largely software agents that comply with the diverse industrial requirements required for the domain they are utilized. Being software agents, a key factor for the industrial acceptance may be the easy and transparent introduction of new and effective algorithms. The latter enable them to operate in heterogeneous and dynamic environments and enhance their collaborative aspects, especially in distributed environments. Agent intelligence may also be reached by embedding self-* algorithms, which properly regulate their behaviors, contributing for the emergence of the desired system characteristics. The latter is seen as a potential promising approach when it comes to dynamic and complex industrial environments. However, decision makers may be hesitant to adopt them, due to the increase of complexity associated to the presence of (distributed) intelligence in industrial systems [5], which in turn may act as a possible road-blocker for the Industrial Agent acceptance.

\section{Hardware}

Industrial agent-based systems comprise of software agents managing, operating, or collaborating with physical hardware devices, being able to implement several paradigms, e.g., holonic [23], [32], CPS [14], bionic, reconfigurable and evolvable production systems, etc. With the continuous empowerment of embedded devices (following Moore's law), agents can now also easily be embedded in these devices as an integral part of them [2], [33]. The integration of physical automation devices (normally tens or hundreds) with the software control system is usually performed on a case-by-case basis, requiring a huge and expensive effort to be implemented, which may be a possible major barrier for the Industrial Agents acceptance [5]. To tackle it, the consideration of methodologies and standard interfaces that support the easy, fast, transparent, and reusable integration of physical automation devices and their functionalities are required. In light of the CPS emergence, hardware considerations that target embedded agents in hardware devices as well as cloud integration may be catalytic [33]. In light of the expected high heterogeneity, the existence of common APIs abstracting from the hardware details but still taking advantage of their capabilities (computation, communication, physical mobility, etc.) may be decisive for the acceptance of industrial agents.

\section{E. Cost}

Cost plays an important role to the adoption of any solution in the industry, and it is multiangled, e.g., includes cost of software development or acquisition, deployment, maintenance and management, personnel training, migration of legacy systems, and hardware acquisition. The required investment to deploy agent-based solutions is usually higher than in traditional solutions [5], [24], in the sense that the adaptation and reconfiguration offered by MAS solutions normally require the presence of flexible and even redundant automation systems. In addition, the other angles of the cost factor may impact the short and mid-term return of investment, which may create a high barrier for the introduction of the technology to the specific industry.

\section{F. Standardization}

Standardization is pointed out by industry experts and also emerges in various surveys [7], [15], [24] as the major key factor that impacts the industrial adoption of the agent technology. The Foundation for Intelligent Physical Agents (FIPAwww.fipa.org) defines a set of standards for the development of MAS [24], focusing especially on the specification of the agents' behaviors and interaction among heterogeneous software agents. However, FIPA is currently not sufficiently addressing many requirements and particularities imposed by industrial environments [34], which may impact the adoption of industrial agent-based systems. The same applies to other related concepts and technologies provided by international standardization organizations, such as World Wide Web Consortium (W3Cwww.w3c.org) and the Organization for the Advancement of Structured Information Standards (OASIS-www.oasisopen.org). However, as key web/Internet standards are being developed in the latter, these could form a basis upon which interoperable and standardized agent solutions can be built. Supporting both key industrial automation and web/Internet technology standards may act as an enabler for Industrial Agent technologies and solutions.

\section{G. Applications}

In the literature, it can be witnessed that the application of agent technology is evident in several domains [2], [35], with scenarios in, e.g., simulation, monitoring, control, reconfiguration, diagnosis, etc. The analysis of a significant number of applications [35] concludes that agent technology may bring tangible benefits to various sectors. The general views in the literature point out that the applicability and expected benefits may depend on the requirements and constraints imposed by the application type. In particular, agents are not seen as suitable for applications requiring hard real-time constraints or just used for a limited scope of tasks with hard real-time tasks constraints 
[24], but can provide benefits in soft real-time applications, such as in production planning and scheduling [23]. It is estimated that only " $30 \%$ of the world's control tasks and about $60 \%$ of the diagnostic tasks would benefit from the application of agentbased techniques" [24]. Therefore, a key factor that may impact the industrial adoption of agent technology is related to actual type of applications that better benefit from the advanced and innovative features provided by agents.

\section{H. Challenges}

As an emergent technology, the success of its industrial adoption is strongly dependent on how well some key challenges and road-blockers can be tackled. For instance, although some benefits of the agent approach can be shown at technical level [27], the proof of tangible business benefits is much more challenging, and often cannot be quantified in advance due to the lack of adequate economic tools and analyses. Industrial Agents require acceptance of their technologies and confidence in their paradigm (e.g., operating in distributed manner), which may be quite difficult to accept in tightly controlled hierarchical industrial settings. Furthermore, although several agent-based systems exist [3], benchmarking approaches that evaluate multiple of these solutions in concrete scenarios are not sufficiently developed, which make difficult to evaluate, compare, and pick the right technologies. The same holds true for methodologies and business models, which have not been adequately addressed with industrial settings in mind. These challenges, seen from the perspective of different players, may be considered as critical issues that should be addressed in the near future to increase the Industrial Agents acceptance.

\section{Approach AND DATA}

The followed approach aims toward identifying and quantifying the potential impact of factors to the Industrial Agent acceptance. As such, the approach can be characterized as a quantitative positivist research [36], and the way its assessment was performed followed the typical approaches in quantitative research field. Having identified the key factors via a literature review, and hypothesized their effects to the Industrial Agent acceptance, the next step comprises of empirical data collection via a survey.

The sample size of this survey is random and comprises a variety of people coming from industry, academia, governmental orgs, etc., with varying degrees of expertise on agents. The survey questions [37] have been constructed with the aim to capture the factors as good as possible, by also taking into account the previous complementary surveys in the area, namely [5], [35]. The collection of data was realized online, where the respondents provided their input in a five-level Likert scale [38] per question. An indicator was providing feedback to the respondents with respect to errors (e.g., forgotten to rate a question) as well as the current percentage of completion for the survey. The data was collected anonymously, with the exception of some demographic data which were explicitly asked. Due to the way, the data collection was done, it was guaranteed that there would be no missing data.
The questions per factor in the survey are encoded as variables, i.e., D1-D8 (Design), T1-T5 (Technology), H1-H4 (Hardware), I1-I5 (Intelligence/Algorithms), C1-C7 (Cost), S1S6 (Standardization), A1-A6 (Application), CH1-CH8 (Challenges), and IAA1-IAA6 (Industrial Agent Acceptance). The empirical data comprises of 118 valid answers $(\mathrm{N}=118)$. All of the variables are measured on the Likert scale, and therefore, there is no reason to exclude variables on skewness unless they exhibit no variance. A kurtosis value in any of the variables that is greater than 1 (or less than -1) may be seen as potentially problematic. However, in practice, only values $>2.2$ (or $<-2.2)$ are problematic [39]. In the dataset, there are some variables that depict kurtosis, however, all of them are less than the practical limits posed (i.e., $[-2,2])$ and, therefore, there is no need to exclude any of them from the dataset, since sufficient variance exists.

As indicated, some demographics on the dataset have been collected. More specifically, it is worth noting that $50 \%$ of respondents came from universities, $24.6 \%$ from industry, $8.5 \%$ from small and medium enterprises, $11 \%$ from research centers, and $3.4 \%$ from nonprofit organizations. With respect to the position, several categories are present, i.e., professors (27.1\%), engineers/developers $(24.6 \%)$, researchers $(18.6 \%)$, managers $(15.3 \%)$, students $(7.6 \%)$, and others $(6.8 \%)$. Most of the respondents (59.3\%) have over ten years of professional expertise, while $30.5 \%$ have four to nine years of expertise, and only $10.2 \%$ are relative inexperienced with one to three years of expertise. Similarly, the years of agent expertise also vary, i.e., $40.7 \%$ have more than ten years of agent expertise, while $40.7 \%$ have four to nine years of agent expertise, and only $18.6 \%$ are relative new to the area with one to three years of agent expertise.

It needs to be pointed out that these demographics overall are not surprising as the survey has been disseminated among the relevant stakeholders of IEEE IES Technical Committee on Industrial Agents as well as relevant conferences, workshops, and agent development mailing lists. As such, the results presented here may depict at large views of the (industrial) agents community.

\section{EMPIRICAL DATA ANALYSIS}

For the analysis of the empirical quantitative data, typical methods are used, i.e., Explorative Factor Analysis (EFA) followed-up by Structural Equation Modeling (SEM) that includes Confirmatory Factor Analysis (CFA) [40]. The aim of carrying out EFA is to investigate if the theoretically considered factors are also evident in the dataset, or if additional ones emerge from the dataset that were not considered. After the cautionary measure of EFA, SEM is applied and the results of the proposed model and the support (or not) of the posed hypotheses is depicted.

\section{A. Explorative Factor Analysis}

The Exploratory Factor Analysis is a multivariate statistics method used to identify the underlying relationships between measured variables [40]. Via the EFA, the aim is to confirm the hypothesis for the factor structure. The Kaiser-Meyer-Olkin is a measure of sampling adequacy and for the overall dataset is 
TABLE I

CRONBACH'S ALPHA PER FACTOR

\begin{tabular}{lcc}
\hline \hline Factor & Variables & Cronbach's $\alpha$ \\
\hline Design & $\{\mathrm{D} 1, \ldots, \mathrm{D} 8\}$ & 0.914 \\
Technology & $\{\mathrm{T} 1, \ldots, \mathrm{T} 5\}$ & 0.811 \\
Intelligence / Algorithms & $\{\mathrm{I} 1, \ldots, \mathrm{I} 5\}$ & 0.857 \\
Standardization & $\{\mathrm{S} 1, \ldots, \mathrm{S} 6\}$ & 0.880 \\
Hardware & $\{\mathrm{H} 1, \ldots, \mathrm{H} 4\}$ & 0.819 \\
Challenges & $\{\mathrm{CH} 1, \ldots, \mathrm{CH} 5, \mathrm{CH} 7, \mathrm{CH} 8\}$ & 0.871 \\
Application & $\{\mathrm{A} 1, \ldots, \mathrm{A} 6\}$ & 0.861 \\
Cost & $\{\mathrm{C} 1, \ldots, \mathrm{C} 6\}$ & 0.847 \\
Industrial Agent Acceptance & $\{$ IAA2, .., IAA6 $\}$ & 0.851 \\
\hline
\end{tabular}

0.696, which is seen as adequate [41]. In addition, the Bartlett's test of sphericity, that tests the null hypothesis that the correlation matrix is an identity matrix, and which would indicate that the factor model is inappropriate is significant; hence, the null hypothesis can be rejected. As such, it is meaningful to proceed with the EFA.

The EFA is conducted using the maximum likelihood as an extraction method and Promax with Kaiser normalization as rotation. The aim is to see if the observed variables load together are correlated and meet the criteria for reliability and validity. A clear loading of variables on factors is witnessed. Hair et al. [42] note that significant factor loadings based on the survey sample size should be approximately 0.5 or higher. It is noted that three variables, i.e., C7, CH6, and IAA1, are below this threshold, and therefore, it was decided to exclude them from further analysis (as also shown in Table I). The nine identified factors explain $57.35 \%$ of the total variance in the empirical data. The factors demonstrate sufficient discriminant validity as the correlation matrix shows no correlations above 0.7 and there are no problematic cross-loadings. For reliability, the Cronbach's $\alpha$ [43] for each factor is calculated, and as shown in Table I, all of them are over 0.8 , which indicates good internal consistency [44].

\section{B. Structural Equation Modeling}

SEM comprises several statistical methods including CFA. The utilization of CFA aims to investigate the fitness of the proposed model (as shown in Fig. 1). Specifically, it is investigated how well the proposed factor structure accounts for the correlations between the variables on the basis of the empirically collected data.

The relative Chi square (CMIN/DF), which is the division of Chi square (CMIN) with the degrees of freedom (DF), is a ratio used to measure the fitness. In this case, the CMIN/DF is 1.256, which indicates a good fit, as only ratios greater than 2 represent an inadequate fit. Other measures of fitness include the Goodness of Fit Index (GFI), and the GFI adjusted for DF (AGFI) [45]. Both GFI and AGFI should be less than or equal to 1 , where a value of 1 indicates a perfect fit. In this case, the GFI is 0.696 and the AGFI is 0.670 , hence, within the acceptance limits. Another fitness measure is the Comparative Fit Index (CFI) [46], which is identical to relative noncentrality index. The CFI is calculated as 0.89 and as it is very near to 0.9 , it indicates an acceptable fit [47]. The root mean square error of

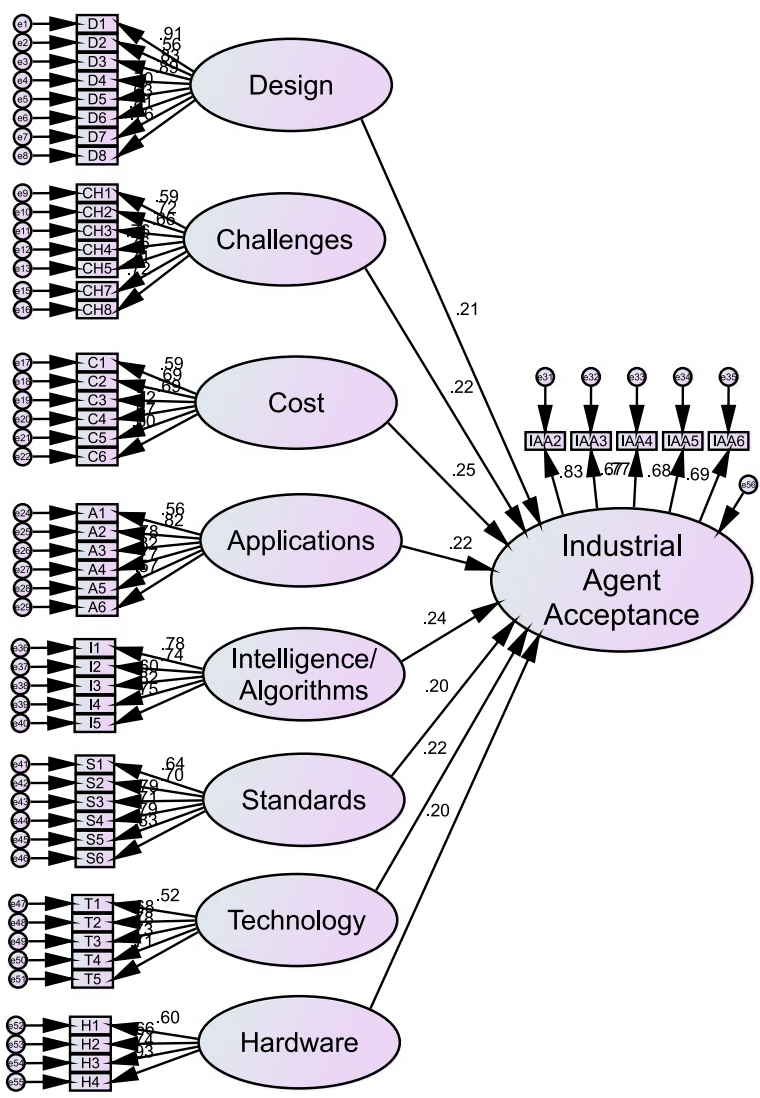

Fig. 2. Structural equation model in AMOS.

TABLE II

TESTING OF HYPOTHESES

\begin{tabular}{lcccc}
\hline \hline Hypothesis & Path & $\begin{array}{c}\text { Path coef } \\
\text { weight }\end{array}$ & $\begin{array}{c}\text { CR value } \\
>1.96\end{array}$ & $\begin{array}{c}\text { Support } \\
\text { Decision }\end{array}$ \\
\hline H1 & Design $\rightarrow$ IAA & 0.211 & 2.391 & Supported \\
H2 & Technology $\rightarrow$ IAA & 0.220 & 2.356 & Supported \\
H3 & Intelligence/Algorithms $\rightarrow$ IAA & 0.243 & 2.650 & Supported \\
H4 & Standardization $\rightarrow$ IAA & 0.196 & 2.172 & Supported \\
H5 & Hardware $\rightarrow$ IAA & 0.198 & 2.201 & Supported \\
H6 & Challenges $\rightarrow$ IAA & 0.220 & 2.410 & Supported \\
H7 & Application $\rightarrow$ IAA & 0.218 & 2.411 & Supported \\
H8 & Cost $\rightarrow$ IAA & 0.248 & 2.680 & Supported \\
\hline \hline
\end{tabular}

approximation (RMSEA) measures the discrepancy between the fitted model and the covariance matrix in the population. In this case, RMSEA is calculated as 0.047 , indicating a good fit [48].

The analysis of the structural equation model is shown in Fig. 2 which shows the AMOS [40] model with all factors and corresponding variables (as summarized also in Table I), as well as the errors. The values on the arrows represent the path coefficients (standardized estimates) which indicate the weight of the links in the path analysis. As it can be seen in Fig. 2, all factors have (as hypothesized $\mathrm{H} 1-\mathrm{H} 8$ ) a positive contribution to the Industrial Agent acceptance.

Table II shows an overview of the hypotheses and the result of SEM. The critical ratio (CR), which is the division of the regression weight estimate, by the estimate of its standard error, and tests for loading significance is also calculated. A CR higher than 
1.96 (or lower than -1.96) indicates two-sided significance at the customary $5 \%$ level. As it can be seen, all of the CR results for all hypotheses are over 1.96 and, therefore, significant. As such, all of the posed hypotheses are supported by the empirical data.

\section{Discussion AND LIMITATIONS}

The acceptance of Industrial Agent solutions is hypothesized to be influenced by several key factors. From the statistical analysis on the collected data, all of the hypotheses $\mathrm{H} 1-\mathrm{H} 8$ (shown in Fig. 1) are supported as their CR value is above the 1.96 limit (shown in Table II). Hence, the empirical data collected via the survey, indicate the support for the theoretical model proposed. Such holistic support may act as a guide for key aspects that need to be addressed if agent-based solutions are to be widely accepted in industry. Some key aspects are pointed out here, while a more qualitative analysis of this survey [37] reveals additional insights.

Design aspects dealing with security, scalability, reliability, robustness, fault-tolerance, openness, modularity, heterogeneity, decentralization, and best practices are seen as key parts. The survey reveals widespread support for these areas with over $80 \%$ of the participants considering them as key aspects. This is in line with the expectations that industrial environments are complex systems that have to work under real-world conditions. In addition, they have to comply not only to a diverse set of landscapes, but be able to deal with emerging industrial needs captured in the mentioned characteristic aspects. A good tackling of design related factors is seen as catalytic toward the wider Industrial Agent acceptance.

Technology plays a key role, and it is evident that a "one-sizefits-all" technology stack is way too limited. As such, support for open contemporary technologies is seen as pivotal. The latter increasingly includes Internet-based technologies that are applied in industrial automation domain. In addition, the usage of modern tools and development environments where Industrial Agent solutions can be developed, deployed, and assessed are needed. The latter are nowadays seen as standard in software-based solutions, and similar support in agents is expected. Hence, the integration with legacy technologies but at the same time openness and flexibility to embrace new ones are seen as catalytic toward increasing the Industrial Agent acceptance.

Intelligence and algorithms enable Industrial Agent solutions to achieve their goals and be effective. As such, these pose as key elements toward the Industrial Agent acceptance. Here, the emphasis is given to the integration of such algorithms in a modular and "plug \& play" approach, as well as to the fact that the needs rely not on monolithic approaches but rather more on the distributed ones that enhance the characteristics of agents, e.g., their collaboration, distributed data processing, and decision-making. Resource efficiency is also pursued, but it comes at a lower priority, probably due to the explosion on the quality and quantity of resources (communication, computation, memory, storage, etc.) that are available to modern industries.

Standardization is seen as overwhelmingly affecting the Industrial Agent acceptance. Standard solutions seem to be preferred and it is believed that industrial automation standards need to be considered and supported. Open Internet and web technologies are seen as the way to go, while admittedly that industry standards for Industrial Agent practices are missing.
Hardware is gaining importance, especially when Industrial Agents are used in the emerging domain of CPS. The support for multiple hardware platforms via common APIs that strike the balance between taking advantage of common hardware capabilities while enabling largely programing of them without dealing with the specificities is needed. Strong bundling to hardware may be needed in some domains, but generally an abstraction level is requested, e.g., in the notion of what the Android OS is doing for the various available mobile devices. Being able to achieve this, it may act as a catalyst toward increasing the Industrial Agent acceptance.

The acceptance of Industrial Agents is bound to some key challenges. All of the investigated challenges should be considered, e.g., business benefits, tools for economic analysis of Industrial Agents, methodologies, business models, better technologies, and benchmarking. It is understood that this area is not adequately investigated and more efforts should be put here as it does impact the (industrial) decision makers view.

The concrete application of Industrial Agents influences also their acceptance. Up to now, the success is mainly in the areas of simulation and emulation of assets/behaviors, and Industrial Agents have successfully been used for monitoring, managing, reconfiguring, and even programing assets. However, real-time control aspects are still work in progress.

Cost tackling is always a sensitive issue and does play a role toward the Industrial Agent acceptance. Any industrially launched solution has to justify its utilization in a way or another in a cost-wise manner in the mid and long term. Costs ought to be seen at multiple levels, out of which cost for hardware, software development, initial deployment, training, and maintenance are seen as critical.

The area of CPS is relevant for Industrial Agents and several efforts have been carried out the last years that demonstrate this link as a survey shows [33]. Key challenges have been identified for CPS [19], and as Industrial Agents already contribute to it [33], the question arises if by increasing the acceptance of Industrial Agents, this could act as an enabler for the CPS domain. As seen in Table III, there are several areas that are common both to 1) the factors that impact Industrial Agent acceptance and 2) the identified challenges for industrial CPS. As such, if Industrial Agents can tackle those issues, they might be able to achieve wider acceptance as they will be in tandem with a key emerging technology, i.e., CPS which are on the roadmap for many diverse industries. The last column in Table III shows, according to authors' view, where agents could meaningfully contribute. It has to explicitly pointed out that Table III does not stem from the empirical data of this paper, and constitutes a point of view by the authors that ought to be seen as potential future research.

Overall, Industrial Agent are seen as a promising approach, which however still needs to be proved in practice. It is believed that Industrial Agents are mature enough for productive utilization and offer added value in productive systems. Also agent-based solutions are seen as being capable of well complementing other approaches, but also many of the Industrial Agent concepts can also be implemented without agent technologies. Especially the high complementarity and substitution may explain the impact on the Industrial Agent acceptance. As all factors contribute to the Industrial Agent acceptance and the 
TABLE III

CPS Challenges [19] TOWARdS Which INDUStRIAL Agents Could Contribute

\begin{tabular}{|c|c|c|c|c|c|}
\hline Area & Key Challenges for Industrial CPS & Difficulty & Priority & Maturity in & IA Contribution \\
\hline \multirow[t]{6}{*}{ CPS Capabilities } & Real-time control of CPS systems & high & high & $4-7$ years & low \\
\hline & Real-time CPS SoS & high & medium & $3-5$ years & medium \\
\hline & Optimization in CPS and their application & high & medium & $4-7$ years & medium \\
\hline & On-CPS advanced analytics & medium & high & $3-5$ years & high \\
\hline & Modularization and servification of CPS & low & high & $3-5$ years & high \\
\hline & Energy efficient CPS & medium & medium & $3-5$ years & low \\
\hline \multirow[t]{3}{*}{ CPS Management } & Lifecycle Management of CPS & medium & medium & $5-8$ years & medium \\
\hline & Management of (very) large scale CPS and CPS-SoS & high & high & $5-8$ years & high \\
\hline & Security and trust management for heterogeneous CPS & high & high & $5-8$ years & low \\
\hline \multirow[t]{5}{*}{ CPS Engineering } & Safe programming and validation of CPS SoS & high & high & $5-10+$ years & low \\
\hline & Resilient risk-mitigating CPS & high & high & $5-10+$ years & low \\
\hline & Methods and tools for CPS lifecycle support & high & high & $3-7$ years & medium \\
\hline & New operating systems and programming languages for CPS and CPS SoS & medium & low & $3-6$ years & low \\
\hline & Simulation of CPS and of CPS-SoS & medium & high & $3-6$ years & high \\
\hline \multirow[t]{5}{*}{ CPS Infrastructures } & Interoperable CPS services & medium & high & $2-5$ years & high \\
\hline & Migration solutions to emerging CPS infrastructures & medium & high & $3-6$ years & high \\
\hline & Integration of heterogeneous/mobile hardware and software technologies in CPS & low & medium & $2-4$ years & high \\
\hline & Provision of ubiquitous CPS services & medium & medium & $3-5$ years & high \\
\hline & Economic impact of CPS Infrastructure & high & high & $3-6$ years & high \\
\hline \multirow[t]{4}{*}{$\overline{C P S}$ Ecosystems } & Autonomic and self-* CPS & high & medium & $7-10+$ years & high \\
\hline & Emergent behavior of CPS & high & medium & $7-10+$ years & high \\
\hline & CPS with Humans in the Loop & high & high & $2-5$ years & high \\
\hline & Collaborative CPS & medium & medium & $5-8$ years & high \\
\hline \multirow[t]{4}{*}{ CPS Information Systems } & Artificial Intelligence in CPS & high & high & $7-10+$ years & high \\
\hline & Cross-domain large-scale information integration to CPS Infrastructures & medium & low & $6-9$ years & high \\
\hline & Transformation of CPS data and information analytics to actionable knowledge & high & high & $4-8$ years & medium \\
\hline & Knowledge-driven decision making/management & high & medium & $6-10+$ years & high \\
\hline
\end{tabular}

critical ratios (as shown in Table II) are very similar, one can hardly prioritize them, and all of them should be considered in any agent solution proposition to industry.

Although this quantitative approach, in which the survey and the empirical data assessment provide key insights on the factors that should be tackled, there are several limitations. From the theory viewpoint, several aspects that had already been identified in the literature including general factors that impact any industrial solutions acceptance, have been considered. These factors are handled at abstract level, but at nature they are more complex and should be investigated individually to get a better understanding of their key components. In addition, there might exist several interdependencies among these factors which are not adequately investigated here. The context of the individual respondents is not fully taken into consideration apart from some general demographic characteristics. However, other aspects, such as geographical and cultural aspects, IT knowledge, programing experience, industry domain, and learning readiness, are not considered. In addition, a larger sampling group, selected via proportional stratified sampling, might be beneficial to derive some more confident results.

\section{CONCLUSION}

Agent technologies have been systematically investigated the last decades, and although several cases of their utilization exist in industry, their breakthrough and wide-scale acceptance is yet to be seen. Several surveys in the literature [4]-[7], [15], [19] point toward some reasons, but mostly from a qualitative view point. In this paper, the focus is on a quantitative methodology.
Having identified the potential factors that impact the Industrial Agent acceptance (considering also the aforementioned surveys), empirical data is collected via a survey, and statistics are applied in order to assess the potential impact of those factors to industrial agent acceptance.

The identified factors considered in this paper are: design, technology, intelligence/algorithms, hardware, cost, standardization, application, and challenges. All of these are found to influence the Industrial Agent acceptance (in a statistically significant way as analyzed). Addressing the discussed challenges is not seen as an easy undertaking. Roadmaps that have been laid out before, dealing with the challenges in (industrial) agent domain, have not really been realized, with several challenges still being open. As such, it is very challenging to provide a concrete roadmap and timelines that can be credibly kept. However, it must be pointed out that there are key elements whose prevalence in the last few years give hope that agents may once more come to the foreground. These elements include the transformation of business to digital business, with the strong interconnection of all stakeholders over Internet-based services as envisioned in Industry 4.0, as well as the ubiquity offered by cloud-based services. In addition, areas that pertain (industrial) agent concepts and technologies including data mining, data management, (artificial) intelligence/machine learning, autonomous CPS, etc., are on a meteoric rise.

With the emergence of CPS, the agent domain may experience a renaissance. The visions for CPS and their applicability in several industrial settings, fit well with the capability of agents and, hence, they could pose a potential interesting approach to implement them. All these gives us a certain degree of confidence, 
that (industrial) agents may yet have another (last?) chance to enter mainstream and achieved the long-promised impact. However, the agent community needs to learn from the failures and successes of the past, and properly tackle the issues related to the factors discussed.

\section{REFERENCES}

[1] M. Wooldridge, An Introduction to Multi-Agent Systems. New York, NY, USA: Wiley, 2002.

[2] P. Leitão and S. Karnouskos, Eds., Industrial Agents: Emerging Applications of Software Agents in Industry. New York, NY, USA: Elsevier, 2015.

[3] T. Jess, P. Woodall, and D. McFarlane, "Evaluating the applicability of multi-agent software for implementing distributed industrial data management approaches," in Studies in Computational Intelligence. New York, NY, USA: Springer, 2015, pp. 199-207.

[4] M. Pěchouček and V. Mařík, "Industrial deployment of multi-agent technologies: Review and selected case studies," Auton. Agent Multi-Agent Syst., vol. 17, no. 3, pp. 397-431, May 2008.

[5] P. Leitão, "Agent-based distributed manufacturing control: A state-ofthe-art survey," Eng. Appl. Artif. Intell., vol. 22, no. 7, pp. 979-991, Oct. 2009.

[6] M. Metzger and G. Polakow, "A survey on applications of agent technology in industrial process control," IEEE Trans. Ind. Informat., vol. 7, no. 4, pp. 570-581, Nov. 2011.

[7] P. Leitão, V. Mařík, and P. Vrba, "Past, present, and future of industrial agent applications," IEEE Trans. Ind. Informat., vol. 9, no. 4, pp. 2360-2372, Nov. 2013.

[8] P. Vrba et al., "A review of agent and service-oriented concepts applied to intelligent energy systems," IEEE Trans. Ind. Informat., vol. 10, no. 3, pp. 1890-1903, Aug. 2014.

[9] V. Vyatkin, "Software engineering in industrial automation: State-of-theart review," IEEE Trans. Ind. Informat., vol. 9, no. 3, pp. 1234-1249, Aug. 2013.

[10] A. Pereira, N. Rodrigues, and P. Leitão, "Deployment of multi-agent systems for industrial applications," in Proc. IEEE 17th Int. Conf. Emerg. Technol. Factory Autom., Sep. 2012, pp. 1-24.

[11] P. Göhner et al., Agentensysteme in der Automatisierungstechnik. Berlin, Germany: Springer, 2013.

[12] M. Luck, P. McBurney, O. Shehory, and S. Willmott, Agent Technology: Computing as Interaction (A Roadmap for Agent Based Computing). AgentLink III, Sep. 2005.

[13] Multi Agent Systems in Industrial Automation, Verein Deutscher Ingenieure (VDI) Verband Der Elektrotechnik Elektronikinformationstechnik (VDE), VDI-Standard: VDI/VDE 2653 Std., Jun. 2010.

[14] A. W. Colombo et al., Eds., Industrial Cloud-Based Cyber-Physical Systems: The IMC-AESOP Approach. New York, NY, USA: Springer, 2014.

[15] V. Mařík and D. McFarlane, "Industrial adoption of Agent-Based technologies," IEEE Intell. Syst, vol. 20, no. 1, pp. 27-35, Jan. 2005.

[16] K. Schild and S. Bussmann, "Self-organization in manufacturing operations," Commun. ACM, vol. 50, no. 12, pp. 74-79, Dec. 2007.

[17] H. Kagermann, W. Wahlster, and J. Helbig, "Securing the future of German manufacturing industry: Recommendations for implementing the strategic initiative INDUSTRIE 4.0," German Nat. Acad. Sci. Eng., Munich, Germany, Tech. Rep., 2013. [Online]. Available: http://goo.gl/HYOfzx

[18] S.-W. Lin et al., "Industrial internet reference architecture," Ind. Internet Consortium (IIC), Needham, MA, USA, Tech. Rep., Jun. 2015. [Online]. Available: http://www.iiconsortium.org/IIRA-1-7-ajs.pdf

[19] P. Leitão, A. W. Colombo, and S. Karnouskos, "Industrial automation based on Cyber-Physical systems technologies: Prototype implementations and challenges," Comput. Ind., vol. 81, pp. 11-25, Sep. 2015.

[20] D. Mcfarlane and S. Bussmann, "Developments in holonic production planning and control," Production Planning Control, vol. 11, no. 6, pp. 522-536, Jan. 2000.

[21] L. Monostori, J. Váncza, and S. Kumara, "Agent-based systems for manufacturing," CIRP Ann. - Manufacturing Technol., vol. 55, no. 2, pp. 697-720, Jan. 2006.

[22] W. Shen, L. Wang, and Q. Hao, "Agent-based distributed manufacturing process planning and scheduling: A state-of-the-art survey," IEEE Trans. Syst., Man, Cybern., C, vol. 36, no. 4, pp. 563-577, Jul. 2006.

[23] R. F. Babiceanu and F. F. Chen, "Development and applications of holonic manufacturing systems: A survey," J. Intell. Manuf., vol. 17, no. 1, pp. 111-131, Feb. 2006.
[24] V. Mařík and J. Lažanský, "Industrial applications of agent technologies," Control Eng. Practice, vol. 15, no. 11, pp. 1364-1380, Nov. 2007.

[25] H. Van Dyke Parunak, "A practitioners' review of industrial agent applications," Auton. Agents Multi-Agent Syst., vol. 3, no. 4, pp. 389-407, 2000.

[26] V. Dignum and F. Dignum, "Designing Agent Systems: State of the practice," Int. J. Agent-Oriented Softw. Eng., vol. 4, no. 3, pp. 224-243, Nov. 2010.

[27] F. Bergenti, M.-P. Gleizes, and F. Zambonelli, Eds., Methodologies and Software Engineering for Agent Systems. New York, NY, USA: Springer, 2004.

[28] "Gartner's 2015 hype cycle for emerging technologies identifies the computing innovations that organizations should monitor," Gartner, Stamford, CT, USA, Aug. 18, 2015. [Online]. Available: http:// www.gartner.com/newsroom/id/3114217

[29] R. A. Belecheanu et al., "Commercial applications of agents," in Proc. 5th Int. Joint Conf. Auton. Agents Multiagent Syst., 2006, pp. 1549-1555.

[30] A. W. Colombo, S. Karnouskos, J. M. Mendes, and P. Leitão, "Industrial agents in the era of service oriented architectures and cloud based industrial infrastructures," in Industrial Agents: Emerging Applications of Software Agents in Industry. New York, NY, USA: Elsevier, Mar. 2015, pp. 67-87.

[31] S. Karnouskos and M. M. J. Tariq, "Using multi-agent systems to simulate dynamic infrastructures populated with large numbers of web service enabled devices," in Proc. Int. Symp. Auton. Decentralized Syst., Athens, Greece, Mar. 2009, pp. 1-7.

[32] S. M. Deen, Ed., Agent Based Manufacturing: Advances in the Holonic Approach. New York, NY, USA: Springer, 2003.

[33] P. Leitão, S. Karnouskos, L. Ribeiro, J. Lee, T. Strasser, and A. W. Colombo, "Smart agents in industrial cyber-physical systems," Proc. IEEE, vol. 104, no. 5, pp. 1086-1101, May 2016.

[34] I. Seixas and P. Leitão, "Standards compliance in industrial agents applications," in Proc. 39th Annu. Conf. IEEE Ind. Electron. Soc., Nov. 2013, pp. 7446-7451.

[35] J. P. Müller and K. Fischer, "Application impact of multi-agent systems and technologies: A survey," in Agent-Oriented Software Engineering. New York, NY, USA: Springer, 2014, pp. 27-53.

[36] D. Straub, M.-C. Boudreau, and D. Gefen, "Validation guidelines for IS positivist research," Commun. Assoc. Inform. Syst., vol. 13, no. 24, pp. 380-427, 2004.

[37] P. Leitão and S. Karnouskos, "A survey on factors that impact industrial agent acceptance," in Industrial Agents: Emerging Applications of Software Agents in Industry. New York, NY, USA: Elsevier, Mar. 2015, pp. $401-429$.

[38] G. Norman, "Likert scales, levels of measurement and the "laws" of statistics," Adv. Health Sci. Educ., vol. 15, no. 5, pp. 625-632, Feb. 2010.

[39] V. A. Sposito, M. L. Hand, and B. Skarpness, "On the efficiency of using the sample kurtosis in selecting optimal $L_{p}$ estimators," Commun. Statist.Simul. Comput., vol. 12, no. 3, pp. 265-272, Jan. 1983.

[40] B. M. Byrne, Structural Equation Modeling With AMOS: Basic Concepts, Applications, and Programming, ser. Multivariate Applications Series, 2nd ed. Oxford, U.K.: Routledge, Taylor \& Francis Group, 2009.

[41] H. F. Kaiser and W. B. Michael, "Little jiffy factor scores and domain validities," Educ. Psychol. Meas., vol. 37, no. 2, pp. 363-365, Jul. 1977.

[42] J. F. Hair, W. C. Black, B. J. Babin, and R. E. Anderson, Multivariate Data Analysis, 7th ed. Englewood Cliffs, NJ, USA: Prentice Hall, 2010.

[43] 1. Cronbach, "Coefficient alpha and the internal structure of tests," Psychometrika, vol. 16, no. 3, pp. 297-334, Sep. 1951.

[44] D. George and P. Mallery, IBM SPSS Statistics 21 Step by Step: A Simple Guide and Reference, 13th ed. Upper Saddle River, NJ, USA: Pearson, 2013.

[45] J. Tanaka and G. Huba, "A fit index for covariance structure models under arbitrary GLS estimation,” Brit. J. Math. Statist. Psychol., vol. 38, no. 2, pp. 197-201, Nov. 1985.

[46] P. M. Bentler, "Comparative fit indexes in structural models," Psychol. Bull., vol. 107, no. 2, pp. 238-246, 1990.

[47] J. C. Loehlin, Latent Variable Models: An Introduction to Factor, Path, and Structural Analysis, vol. 12, 3rd ed. Mahwah, NJ, USA: Lawrence Erlbaum Associates Publishers, 1998, no. 4.

[48] R. C. MacCallum, M. W. Browne, and H. M. Sugawara, "Power analysis and determination of sample size for covariance structure modeling," Psychol. Methods, vol. 1, no. 2, pp. 130-149, 1996.

Authors' photographs and biographies not available at the time of publication. 